\title{
Población flotante y ciudad desde una perspectiva socioespacial: revisión de estudios recientes*
}

\section{The floating population and the city from a socio-spatial perspective: A review of recent studies}

\author{
Carlos Gándara Woongg** \\ Fernando Padilla Lozano*** \\ Pablo Gutiérrez Castorena****
}

Universidad Autónoma de Aguascalientes (UAA), Aguascalientes, México.

\begin{abstract}
Resumen
La población flotante como fenómeno social tiene un impacto directo en las ciudades por las que transitan. Al ser un hecho la creciente presencia de estos pobladores en las urbes contemporáneas, es necesario realizar un estudio que abarque el impacto de dicha población en la ciudad y en el desarrollo urbano de esta. Basado en el examen de cuarenta
\end{abstract}

* Este artículo forma parte de la investigación doctoral del primer autor, estudio que fue financiado por el Consejo Nacional de Ciencia y Tecnología (Conacyt) de México. Los autores desean expresar su sincero agradecimiento al Centro de Ciencias del Diseño y de la Construcción de la Universidad Autónoma de Aguascalientes, así como al Conacyt por el apoyo financiero, y al Dr. Jesús Pacheco Martínez por su amable ayuda en la corrección de la primera versión de este artículo.

** Maestro en Arquitectura, Universidad Autónoma de Baja California (UABC). Becario doctoral Conacyt, doctorado en Ciencias de los Ámbitos Antrópicos. Docente de la Facultad de Arquitectura y Diseño, UABC. Correo electrónico: carlos_gw7@ hotmail.com

*** Doctor en Ciencias Antropológicas, Universidad Autónoma Metropolitana (UAM). Profesor investigador de tiempo completo, Departamento de Sociología y Antropología, Universidad Autónoma de Aguascalientes (UAA). Correo electrónico: fpadill1@ correo.uaa.mx

**** Doctor en Ciencias Sociales y Humanidades, Universidad Autónoma de Aguascalientes (UAA). Profesor investigador de tiempo completo, Departamento de Sociología y Antropología, UAA. Correo electrónico: pgutier@correo.uaa.mx

Cómo citar este artículo: Gándara, C.; Padilla, F. y Gutiérrez, P. (2020). Población flotante y ciudad desde una perspectiva socioespacial: revisión de estudios recientes. Si Somos Americanos. Revista de Estudios Transfronterizos, 20(1), 103-122. doi: 10.4067/S0719-09482020000100103 
artículos, este trabajo presenta una revisión crítica e integradora de los documentos realizados sobre la población flotante en el período de 1990 a 2019. La revisión bibliográfica orienta el enfoque socioespacial al incluir a este segmento poblacional y su correlación con la ciudad en términos sociales y espaciales, entendiendo la ciudad como el espacio donde se desarrollan las interacciones sociales. Los resultados reflejan los hallazgos y limitantes de un problema con diferentes variantes, que cambian dependiendo del contexto local, regional y global, así como se entregan recomendaciones para futuras investigaciones.

Palabras clave: población flotante, ciudad, movilidad.

\begin{abstract}
The floating population as a social phenomenon has a direct impact on the cities through which its members pass. Given its increasing presence in contemporary urban settings, there is a need to ascertain the impact of the floating population on the city and its urban development. Based on a review of 40 articles, this paper presents a critical and integrative analysis of papers published on the floating population for the period spanning 1990 to 2019 . The literature review guides the socio-spatial focus by including this population segment and its correlation with the city in social and spatial terms, understanding the city as the space in which social interactions take place. The results reflect the findings and limitations of an issue that has different variants, and which changes depending on the local, regional and global context. Recommendations for future research are also included.
\end{abstract}

Keywords: floating population, city, mobility.

\title{
Introducción
}

La movilidad humana juega un papel preponderante en la dinámica de crecimiento de las ciudades. Hoy en día estos flujos se alimentan de diversas realidades sociales, así como de dimensiones temporales y espaciales que conducen al surgimiento del estudio de la población flotante. Esta cobra relevancia en la sociedad actual cada vez más globalizada y en ciudades marcadas por la industrialización (Liang y Ma, 2004). Realizar una revisión bibliográfica de la población flotante demanda, en primer lugar, establecer un concepto que delimite su magnitud. Esto, debido a que las dimensiones, alcances y actores varían entre autores, países e instituciones. Por lo anterior, para esta búsqueda se entenderá como población flotante a aquellos que viajan de un lugar a otro y se encuentran en un lugar en 
determinado momento, independientemente del tiempo que llevan viviendo ahí, pero cuya residencia habitual no es dicho lugar (Garrocho, 2011).

Los artículos realizados al respecto se encuentran dispersos en diferentes áreas del conocimiento, como: geografía, demografía, sociología, economía, urbanismo y política, destacándose la geografía y el urbanismo, disciplinas que han abordado la complejidad de determinar la cuantía e impacto de estas personas en un territorio determinado. Dentro de este universo, este trabajo es el resultado de una búsqueda bibliográfica que antecede a un proyecto de investigación, que trata de descifrar el impacto de los pobladores flotantes en la ciudad contemporánea, por lo que contiene un enfoque socioespacial.

Por lo anterior, es necesario partir del hecho de la existencia de reglas no siempre escritas que norman la relación entre las personas y el territorio, y que a su vez estructuran la conducta de los individuos en el espacio urbano (Duhau y Giglia, 2008). Es por eso que la relación sociedad y espacio es un todo indisoluble a causa de una relación bidireccional, donde lo social altera lo espacial y lo espacial subordina lo social. No obstante, este vínculo no es espontáneo puesto que implica un proceso de apropiación y adaptación de ambos, y es allí, precisamente, donde se entiende la importancia de descifrar las prácticas socioespaciales como base para entender y regular el uso y ocupación de los espacios, y por ende, la construcción social circundante de los mismos (Quintero, 2008). Dado que el espacio apropiado implica en esencia una identidad cultural con el entorno, generada por la inserción física directa, ya sea porque el individuo ha nacido en esa ciudad o la habita desde algún tiempo conviviendo en el área y con sus ocupantes, produce una interacción de la cual resulta la relación natural con el territorio y sus pobladores, incorporada al imaginario social (Teixeira, 2000).

Dicho lo anterior es necesario reconocer que la situación socioespacial de los pobladores flotantes requiere de un entendimiento de su realidad como usuario del espacio, así como de las características morfológicas y sociales.

Este artículo se inicia con el análisis de la definición de población flotante y su relación con la ciudad, seguida de la metodología de investigación. Los artículos seleccionados se separaron en dos secciones: la primera aborda la dimensión social de la población flotante, como las conductas de los residentes que conforman esta y su interacción con los residentes permanentes; y la segunda está enfocada en el uso del territorio con el fin de entender la forma en que los residentes temporales habitan la ciudad y el papel que juegan estos en el desarrollo urbano. Por último, la discusión incluye un resumen de los hallazgos de este estudio y recomendaciones para futuras investigaciones relacionadas con el tema. 


\section{El poblador flotante en la sociedad actual}

Antes de profundizar en el tema específico de la población flotante es necesario comprender al hombre contemporáneo en general y cómo los fenómenos sociales de décadas pasadas han influido en la construcción social de hoy en día. Resulta pertinente retomar visiones filosóficas sobre el hombre, como la del antropólogo italiano Ernesto de Martino (1977), para quien el concepto de presencia va más allá del estar localizado en el espacio físico específico, puesto que es necesario el estar consciente en el tiempo, en el sentido filosófico de "estar presente en la historia" mediante la cultura. Parafraseando a Webber (1963), las ciudades son el espacio construido por el hombre, máxima expresión de su cultura y, de forma particular, de los defensores de la cultura moderna, de la cual deviene lo que identificamos como el cadáver al que alude Duke (2007): el humanismo, cuya connotación remite directamente a una acción derivada o asociada al hombre, lo cual, visto así, suena redundante, pues lo humano es intrínseco al hombre y en pocas ocasiones al sujeto. El primero es mayormente una figura filosófica, mientras el segundo (al igual que el individuo) adquiere categoría sociológica.

Habitamos, convivimos y nos relacionamos modificando el espacio, transformando el territorio y reconstruyendo nuestro entorno. El medio deja de ser natural, un elevado porcentaje del planeta se encuentra intervenido o afectado por el ser humano. Adaptamos el espacio y el territorio a nuestra necesidad y requerimientos, todo en beneficio del hombre, con el argumento de elevar la calidad de vida, contar con mayor educación, servicios e infraestructura para la salud o el ocio.

Para descifrar al hombre actual y sus prospectivas es imprescindible ampliar la visión del tiempo y espacio para dejar de concebirlo como algo fijo, para de esta manera dar cabida a un sinnúmero de expresiones sociales que de diferentes maneras buscan encontrar la libertad de ataduras físicas y psicológicas.

De la misma manera que el hombre, la ciudad contemporánea presenta retos para su correcto desarrollo. Es por eso que el análisis de la población flotante surge de los estudios urbanos y de la evolución de la ciudad frente a los fenómenos de la urbanización, la metropolización con la generación de grandes poblaciones marginales no incorporadas a los servicios urbanos y a la posesión de una vivienda decorosa y, últimamente, también se aplica a la población que como consecuencia de la globalización y la incorporación de la informática genera cambios en los tipos de población que comparten transitoriamente la ciudad, sin residir en ella (Panaia, 2010).

En esta exploración ciertos segmentos de la población se han encaminado a un proceso de emancipación del espacio físico, lo que conlleva a diferentes fenómenos de movilidad. Dentro de esta movilidad existe un grupo que rompe con el paradigma de permanecer en un lugar y echar raíces, lo que incrementa el número de posibles lugares donde habitar. 
Este grupo se desplaza por diferentes regiones conforme las condiciones vayan cambiando y resulten favorecedoras, sin arraigarse en un lugar determinado, convirtiéndose así en población flotante.

Bell y Ward (2000) evidencian las principales diferencias entre migrantes y población flotante, conceptos que a primera vista podrían considerarse como uno solo; sin embargo, guardan diferencias, por lo que se hace necesario manejar claramente los dos conceptos por separado. Como muestra el Cuadro $\mathrm{N}^{\circ} 1$, las principales diferencias entre pobladores flotantes y migrantes radican en el objetivo, temporalidad, retorno, duración y frecuencia, no obstante, comparten la escala y motivación. Además, fue preciso incorporar en este cuadro a los residentes permanentes para tener a todos los grupos que conforman la población de un territorio, junto con considerar otros elementos como motivación, escala y la definición misma de cada tipología de habitante de una urbe.

Cuadro $N^{\circ} 1$ : Diferencias entre residente, migrante y flotante

\begin{tabular}{llll}
\hline Población & Residente & Migrante & Flotante \\
\hline Definición & $\begin{array}{l}\text { Sin intención de } \\
\text { cambio }\end{array}$ & $\begin{array}{l}\text { Cambio permanente de } \\
\text { residencia habitual }\end{array}$ & Cambio no permanente \\
\hline Objetivo & Permanencia & Arraigarse & Residencia temporal \\
\hline Temporalidad & Vitalicia & Indefinida & $\begin{array}{l}\text { Definida en la mayoría de } \\
\text { los casos }\end{array}$ \\
\hline Retorno & N/A & No hay intención & $\begin{array}{l}\text { Existe la intención de } \\
\text { regresar al hogar }\end{array}$ \\
\hline Duración & Permanente & Ultima relocalización & Variable \\
\hline Frecuencia & N/A & Una sola & $\begin{array}{l}\text { Varían dependiendo de } \\
\text { cada situación }\end{array}$ \\
\hline Escala & Local & $\begin{array}{l}\text { Regional, nacional e } \\
\text { Internacional }\end{array}$ & $\begin{array}{l}\text { Regional, nacional e } \\
\text { Internacional }\end{array}$ \\
\hline Motivación & Productivas y & $\begin{array}{l}\text { Productivas y } \\
\text { reproductivas }\end{array}$ & $\begin{array}{l}\text { Productivas y } \\
\text { reproductivas }\end{array}$ \\
\hline
\end{tabular}

Fuente: elaboración propia basada en Bell y Ward (2000).

Entre los conceptos de mayor aceptación en las publicaciones que abordan la población flotante está la definición de Goodkind y West (2002), que se refieren a este grupo demográfico como personas sin residencia permanente o simplemente a diversos subgrupos demográficos sin un lugar de residencia definido; sin embargo, algunos estudios adaptan la definición dependiendo de los resultados que desean obtener e incluyen pocas variantes, lo que le otorga un cierto grado de ambigüedad al concepto. 
La población flotante y la expansión urbana son dos cuestiones centrales en el proceso de urbanización global (Luo, Zhang, Wu, Shen, J., Shen, y Xing, 2018). Su cuantificación no es posible si el Estado no realiza registros sobre cambios residenciales y genera con ello estadísticas, por lo que se requiere de instrumentos de medición que proporcionen la información necesaria para de esta manera responder a las demandas de recursos locales (Goodkind y West, 2002). La población flotante es una parte importante del motor económico de una sociedad y juega múltiples roles en la urbanización (Luo et al., 2018). Es por esta razón que existe un vínculo directo entre estas personas y la ciudad, a pesar de que su estancia sea solo temporal. Como se aprecia en la Figura $\mathrm{N}^{\circ} 1$, su presencia tiene un impacto directo en diferentes tópicos socioespaciales de toda urbe.

Figura $N^{\circ} 1$ : Tópicos socioespaciales que se ven impactados por las poblaciones flotantes

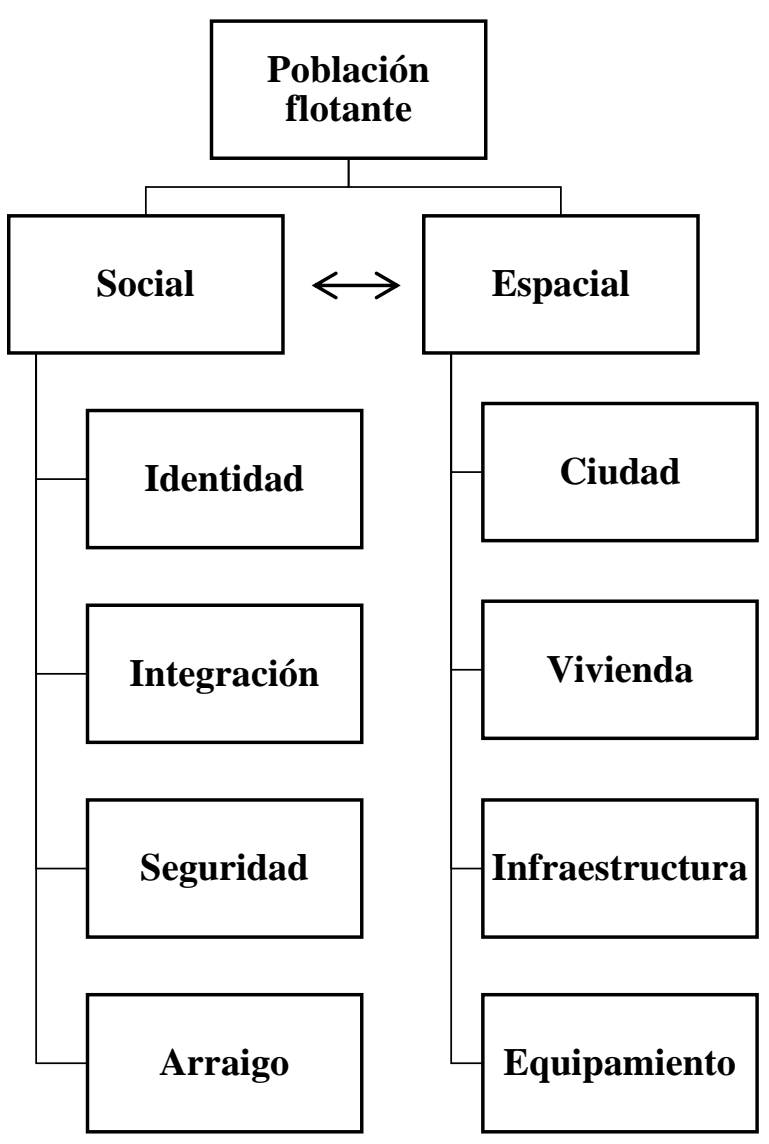

Fuente: elaboración propia. 


\section{Metodología}

\section{Recuperación de documentos}

La estrategia de búsqueda se basó en las palabras clave, población flotante y migración flotante, que son términos utilizados en diferentes países y por diversos autores para referirse al mismo segmento poblacional; seguidas de vocablos como ciudad, social o espacial, con el fin de evitar textos que no tuviesen relación con el enfoque socioespacial.

El proceso de selección se basó en la siguiente estrategia de trabajo:

i. Las bases de datos consideradas fueron: EBSCO, Springer, Scopus, ScienceDirect. De estos se seleccionó EBSCO, por ser el que arrojó el mayor número de resultados: 195.

ii. El rango de tiempo abarcó desde 1990 hasta 2018/04/19. Se consideró el concepto en inglés, floating population. Se tomaron en cuenta solo artículos de revistas académicas.

iii. Se eliminó los artículos que, aunque contenían algunas de las palabras clave, no correspondían a algún área de estudio deseado, como: biología, zoología, ingeniería, astrología, salud, etc. Este criterio determinó la cantidad de 60 artículos.

iv. Se leyó detenidamente el resumen de cada artículo y se eliminaron los que fueron irrelevantes. Finalmente el número se redujo a 40 artículos para la revisión.

\section{Resultados generales}

Los primeros resultados generados por la revisión general de los 40 artículos seleccionados mostraron lo que se describe a continuación. Como se observa en el Cuadro $\mathrm{N}^{\circ} 2$, los artículos se obtuvieron de 26 revistas diferentes, de las que despuntó Habitat International con ocho artículos. Las disciplinas fueron diversas, predominando: geografía y urbanismo. El Gráfico $\mathrm{N}^{\circ} 1$ muestra que el número de artículos relevantes publicados entre $1990 \mathrm{y}$ 2018 se concentra en las últimas dos décadas, lo que indica que los estudios que utilizan el termino población flotante son relativamente nuevos. El Gráfico $\mathrm{N}^{\circ} 2$ exhibe la distribución de los 40 trabajos en las distintas regiones y países del mundo, lo que permite observar que en su mayoría son generados en China. Esto evidencia que el término aún no es de uso común en todo el mundo, no obstante, con otros métodos de búsqueda se encontró que, en países como España, Australia y México, el término empieza a cobrar fuerza, por lo que se espera contar con información al respecto en el futuro. 


\section{Cuadro $N^{\circ}$ 2: Resumen de los trabajos seleccionados y las revistas}

\begin{tabular}{|c|c|c|}
\hline Disciplina & \begin{tabular}{|l} 
Título de la revista \\
\end{tabular} & No. Artículos \\
\hline Urbanismo & International Journal of Urban \& Regional Research & 1 \\
\hline Geografía & Habitat International & 8 \\
\hline Urbanismo & Urban Studies & 2 \\
\hline Demografía & Population \& Development Review & 1 \\
\hline Demografía & Population \& Environment & 1 \\
\hline Urbanismo & Urban Studies & 2 \\
\hline Geografía & Journal of Contemporary Asia & 1 \\
\hline Migración & International Migration Review & 2 \\
\hline Demografía & Population \& Development Review & 1 \\
\hline Política & Social Policy \& Administration & 1 \\
\hline Antropología & Ethnic \& Racial Studies & 1 \\
\hline Interdisciplinaria & Plos ONE & 1 \\
\hline Geografía & Applied Geography & 2 \\
\hline Antropología & Critique of Anthropology & 1 \\
\hline Urbanismo & Cities & 2 \\
\hline Vivienda & Housing Studies & 1 \\
\hline Geografía & Chinese Geographical Science & 1 \\
\hline Geografía & Journal of Contemporary Asia & 1 \\
\hline Salud & Health Promotion International & 1 \\
\hline Ecología & Ecological Indicators & 1 \\
\hline Ecología & Ecological Modelling & 1 \\
\hline Geografía & Eurasian Geography \& Economics & 1 \\
\hline Economía & Journal of Socio-Economics & 1 \\
\hline Geografía & Asia Pacific Viewpoint & 2 \\
\hline Geografía & Social \& Cultural Geography & 1 \\
\hline \multirow[t]{2}{*}{ Geografía } & Modern China & 2 \\
\hline & Total & 40 \\
\hline
\end{tabular}




\section{Gráfico $N^{\circ}$ 1: Artículos publicados por cada década}

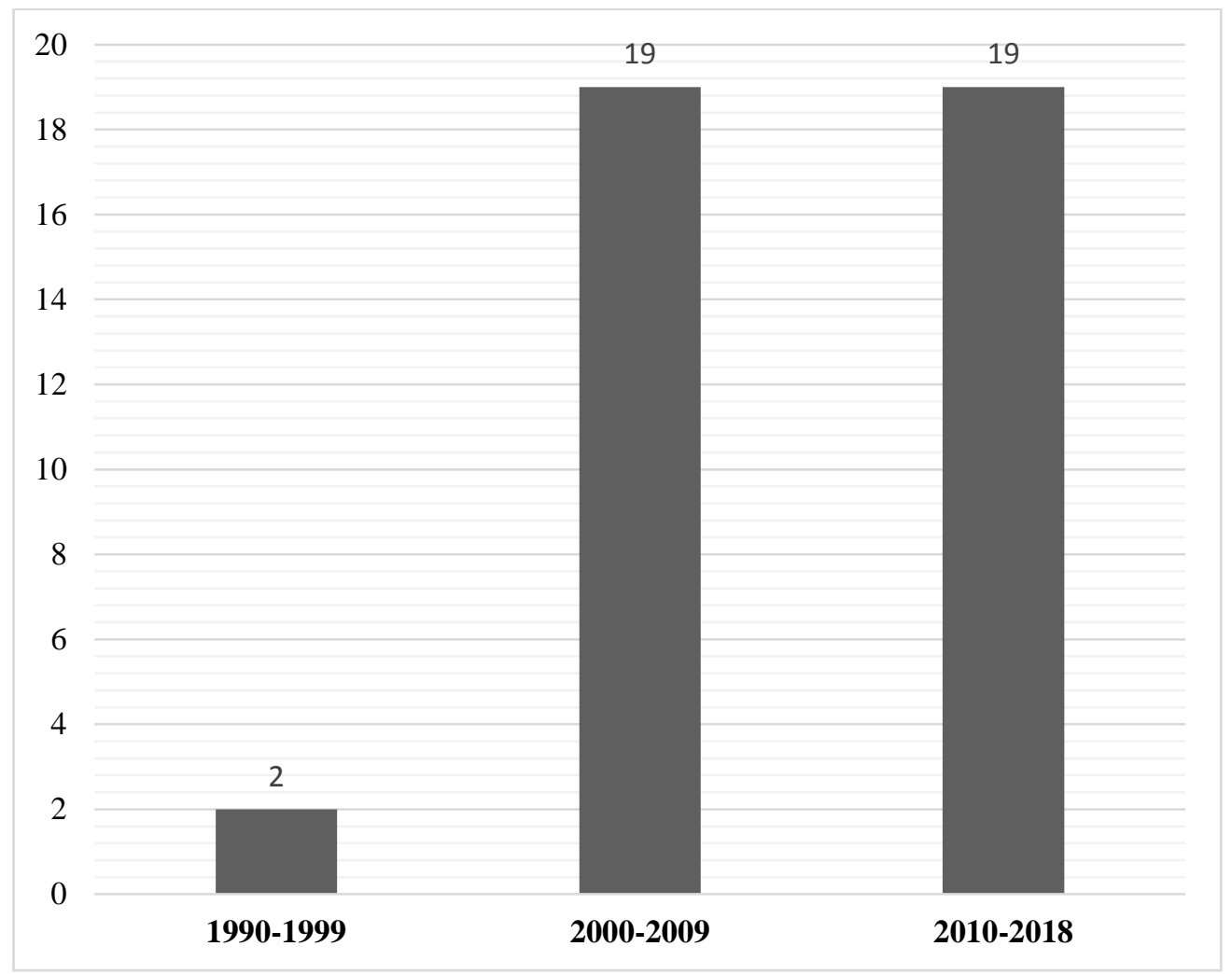

Fuente: elaboración propia.

Gráfico $N^{\circ}$ 2: Artículos por país

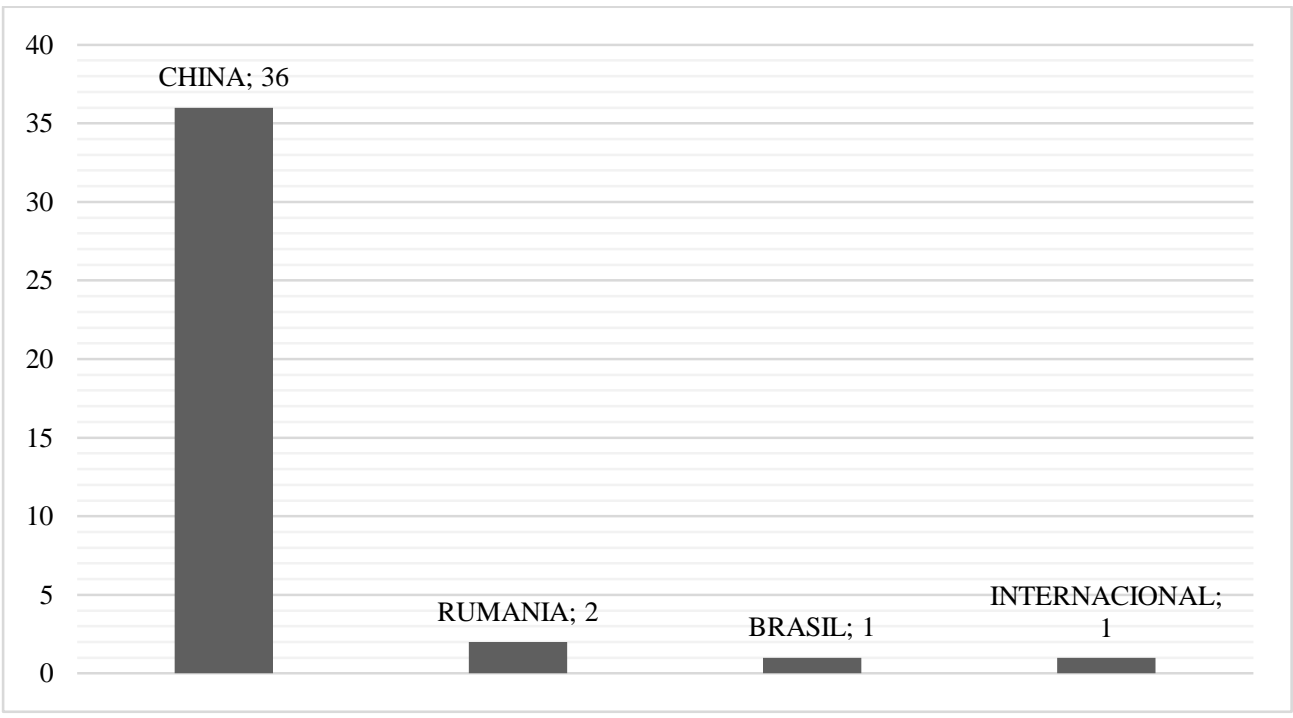

Fuente: elaboración propia. 


\section{Revisión de artículos con temáticas socioespaciales ligadas a la población flotante}

Desde inicios del siglo XXI, la población flotante ha aumentado de manera continua y rápida con un complejo mecanismo de formación. Investigaciones han demostrado que el rápido crecimiento de la riqueza es la causa principal del movimiento poblacional y que el desequilibrio económico determina la dirección de los desplazamientos en gran medida (Shen, 2013; Zhu, 2007). Similar al comportamiento de la población flotante en los países occidentales prósperos, en los países subdesarrollados también es impulsado por las oportunidades de trabajo (Qiao, Li, Liu, y Yang, 2016). Para lograr un mayor entendimiento de estos flujos, Meeus (2012) sugiere mezclar o triangular métodos cualitativos y cuantitativos para contextualizar la investigación de las poblaciones flotantes, ya que esto ofrece una solución al problema del espacio-tiempo, que son las dos variables que intervienen.

Las ciencias sociales identificaron tres principales razones para investigar el tema de las poblaciones flotantes. Primero, los migrantes flotantes no tienen el mismo acceso a beneficios que están reservados para los residentes locales, como: ciertos puestos de trabajo, atención médica e instituciones educativas. En segundo lugar, gran parte de esta población resulta tradicionalmente del desplazamiento desde áreas rurales hacia urbanas en respuesta a la creciente demanda de mano de obra barata, particularmente en las regiones de rápida industrialización. En tercer lugar, la población flotante desempeña un papel vital para el progreso de su ciudad de origen, pues estos migrantes envían remesas considerables desde el extranjero a sus familiares (Zai y Zhongdong, 2004). A continuación, se describen algunas de las principales categorías analíticas y dimensiones que desarrollan los artículos seleccionados.

\section{Arraigo}

La idea de arraigo que pudieran desarrollar los pobladores flotantes para abandonar su situación refiere al hecho de afincarse de modo permanente o echar raíces en algún lugar. En el estudio de Zhu (2007) se concluye que la intención de asentarse de la población flotante en las ciudades no es tan fuerte como se puede suponer; de hecho, permanecer circulando o "flotando" sigue siendo la elección de la mayoría. Según el estudio cualitativo realizado por Mohabir, Jiang, y Ma (2017) sobre la población flotante, estos investigadores sugieren que, respecto a la determinación de arraigarse de los pobladores flotantes en un determinado lugar, no solo cuentan factores económicos como la integración al mercado laboral y el poder adquisitivo, sino que tal decisión está determinada por elementos que van más allá de lo económico; factores sutiles como objetivos educativos para los hijos, la identidad o el sentido pertenencia al lugar, pueden ser determinantes. Por lo anterior se 
pude deducir que existe una clara diferencia entre los migrantes y los pobladores flotantes en relación con la meta de asentarse en un lugar específico.

\section{Segregación}

Al estudiar a la población flotante se pueden encontrar algunas disparidades que se evidencian en sus conductas sociales dentro de la ciudad. Estas actitudes se reflejan en las interacciones diarias entre los residentes temporales y permanentes, por lo que debería ser del interés del Estado incorporar disposiciones para mejorar las interacciones sociales entre ambos grupos, confiando en la sociedad civil para que esta marque las pautas (Bingqin, 2006). Los programas de inserción deben estar dirigidos a la integración social y espacial de los niños urbanos y flotantes, con la visión de promover amistades intergrupales que prevengan el desarrollo de prejuicios, de manera que esta sea la base sobre la cual cerrar la brecha entre los residentes y la población flotante (Nielsen, Nyland, Smyth, Mingqiong y Jiuhua Zhu, 2006).

\section{Seguridad}

Según el estudio realizado por Caminha et al. (2017) en varias ciudades de Brasil, estos infieren que a mayor flujo de pobladores flotantes en un área particular de la ciudad tendrá lugar una tasa proporcionalmente mayor de delitos contra la propiedad de la población residente. En los casos en que la población flotante supera proporcionalmente a los residentes, se observa un aumento de delitos graves. Este resultado muestra que el efecto se debe correlacionar con la población residente o flotante, según el tipo de crimen. Cabe destacar que este fue el único artículo encontrado que aborda el tema de seguridad, por lo que sería relevante realizar el mismo estudio en diferentes regiones del mundo para poder llegar a conclusiones certeras y no estigmatizar a este segmento de la población.

\section{Identidad}

Según Côtè y Levine (2002), la identidad se compone de tres planos: uno social que posiciona al individuo dentro de la estructura social; otro personal que cubre los aspectos de la experiencia individual que surgen de las interacciones, que contribuyen a conformar un conjunto de elementos que una persona utiliza para describirse a sí misma (autoconcepto); y, por último, el Yo que proporciona el sentido subjetivo de continuidad. De igual manera, el sentido de pertenencia juega un papel relevante del que no quedan exentos los pobladores flotantes solo por su presencia efímera en la ciudad, puesto que 
consiste en la implicación personal en un sistema social, de tal forma que la persona siente que es una parte indispensable e integral de ese sistema (Anant, 1966).

Al ser un grupo poblacional tan diverso y cambiante, la población flotante tiende a encontrarse en constante conflicto con el conjunto de rasgos o características que les permite distinguirse del resto. Según Ji-Wei et al. (2010), a miembros de una población flotante les resulta igual de complicado mantener tanto la identidad y el sentido de pertenencia a su ciudad natal, como la adaptación a una ciudad anfitriona, por lo que luchan por instalarse en la vida de la ciudad anfitriona y mantener nexos con sus lugares de origen. Para descifrar el tema de la identidad, se tiene que tener en cuenta el tiempo que el poblador temporal pasa fuera de su lugar de origen, de ahí que Tan et al. (2008) consideran como población flotante a los habitantes que residen en una ciudad por seis meses al año.

Otro aspecto atendible es el ambiental. Al respecto, Gu y Ma (2013) reflexionan que este se ve influido por estos actores, puesto que la falta de sentido de pertenencia, por parte de la población flotante, resulta en una carente atención al entorno local y al control de la contaminación. Esto debilitaría la conciencia ambiental, teniendo incluso más influencia que la que pudieran desarrollar los habitantes permanentes.

\section{Ciudad}

El espacio urbano que comprende a la ciudad, consiste en una serie de sumas y restas sucesivas que refleja en sí mismo la propia historia de su construcción (Ley y Fimbres, 2011). Hoy en día, las ciudades tanto de países desarrollados como de las en vías de desarrollo se enfrentan al desafío del crecimiento sustentable de sus urbes, las que deben no solamente desarrollarse organizadamente sino de manera integral. En el estudio de Wu, Luo, Zhang y Skitmore (2016), se afirma que entre los puntos a considerar para lograr el desarrollo sustentable se debe tomar en cuenta a la población flotante. Esto, porque la mayor concentración de estos pobladores flotantes está en las grandes ciudades. Debido a la presencia de este grupo poblacional empiezan a emerger nuevos patrones de urbanización. Walcott y Pannell (2006) sostienen que actualmente no se cuenta con algún mecanismo que permitan medir su influencia. De haber información suficiente, se podría considerar y usar para la correcta planeación urbana.

\section{Vivienda}

La vivienda es parte crucial en la estructura de la ciudad, al ser la edificación que cubre las necesidades básicas de la población. En el caso de la población flotante, el tema de la vivienda resulta aún más distintivo, puesto que los integrantes de estos grupos flotantes no 
tienen un vínculo con el resto de la urbe. Una particularidad de estos grupos es que al estar limitados por el acceso a los recursos públicos y reconocerse como un grupo socialmente aislado, interactúan en mayor medida con los vecinos, quienes probablemente están en la misma categoría de población flotante. Esto se refleja en la propensión de ayudar al otro poblador flotante, esperando que de alguna esta actitud resulte en una acción recíproca que se transforme en una red de ayuda. De ahí que la elección del vecindario para habitar una vivienda sea una decisión de suma importancia para ellos (Wu y Logan, 2016).

Las residencias de la población flotante se dividen en cinco tipos: familia y hogares colectivos; posadas y hoteles; medios de trasporte (barcos, automóviles, vagones de tren etc.); empresas e institutos; gratuitos: mercados, estaciones ferroviarias y de autobuses, y otros lugares donde los migrantes y los transeúntes se congregan temporalmente (Roberts, 2002). Estudios han revelado que la población flotante urbana (en particular, aquella compuesta por quienes emigran de la zona rural a la urbana) paga un precio mucho más alto por la vivienda que los residentes permanentes, incluso aunque su espacio de vida sea más reducido que la de estos.

Según el estudio "Condiciones de vida de la población flotante urbana en China urbana" de Jiang (2006), los pobladores flotantes en la mayoría de las ocasiones viven en colectivos en mal estado o incluso en sus lugares de trabajo; sin embargo, en muchos de los casos las viviendas que habitan presentan mejores condiciones y servicios que las de su lugar de origen. Otro dato lo aportan Logan, Fang y Zhang (2009), quienes señalan que las poblaciones flotantes son más propensas a estar en viviendas de alquiler y que es menos probable que construyan sus casas propias en comparación con las personas más arraigadas localmente. Estudios previos han encontrado correlaciones entre la población flotante y los precios de la vivienda, debido al limitado acceso y a un aumento en la demanda, concluyendo que, cuanto mayor sea la proporción de la población flotante en una ciudad, mayores son los precios (Wang, et al., 2017).

En general, las condiciones de vivienda de esta población son precarias y temporales (Shen y Huang, 2003). La satisfacción residencial de los pobladores flotantes no sigue patrones estándares. La mayoría de estos expresan una opinión neutral o buena acerca de su residencia, a pesar del hecho de que viven hacinados y en edificaciones en malas condiciones, por lo que se debería prestar mayor atención a la mejora de las áreas de vivienda y al entorno de la comunidad, para que estas residencias proporcionen el espacio que contribuya a la rápida adaptación a la nueva forma de vida (Tao, Wong y Hui, 2014). 


\section{Infraestructura}

El transporte tiene un estrecho nexo con la ciudad y los pobladores flotantes, ya que cuanto más rápido es el desarrollo urbano y el de los medios de transporte de una ciudad, mayor será la velocidad con la que llegue la población flotante (Yue et al., 2005). Esto queda evidenciado en un segmento pocas veces considerado, que son los turistas. Estos solo están presentes en las ciudades a las que se tiene fácil acceso (Crang y Zhang, 2012); por lo tanto, en todo estudio sobre estos pobladores se debe de tener en cuenta los medios de transporte que utilizan.

\section{Discusión}

Solo analizando la complejidad de esta interacción social y espacial, así como la evaluación de la situación pasada, presente y futura de la población flotante, se podría proponer soluciones y estrategias para la inserción urbana de estos moradores temporales. Como se espera que los académicos continúen investigando al respecto, esta sección discute algunas posibles direcciones de investigación.

\section{Tópicos sociales}

La sociedad en general se encuentra en un constante cambio y la población flotante solo es el resultado de esta transformación. La principal diferencia entre los migrantes y los pobladores flotantes es el arraigo. Esto debido a que la meta de un migrante es establecerse en su destino, mientras que los otros prefieren seguir en movimiento (Mohabir, Jiang y Ma, 2017; Zhu, 2007). La interacción entre residentes temporales y permanentes es un tema crucial para la correcta inserción social. Diversos investigadores (Bingqin, 2006; Nielsen et al., 2006) abordan esta problemática, estableciendo recomendaciones que deberían ser consideradas por las autoridades. El tema que presenta el mayor abandono es el de la seguridad, ya que solamente se encontró un estudio al respecto, localizado en Brasil, tal como antes se comentó. Este tópico resulta crítico para toda comunidad, sobre todo para los residentes, quienes podrían desarrollar algún tipo de prejuicio que estigmatizaría a las personas foráneas. Como toda evolución no sucede de golpe, esta se va dando paulatinamente, lo que se evidencia en la identidad de los pobladores temporales, quienes según Ji-Wei et al. (2010) entran en conflicto al tratar de adaptarse a la ciudad, pues les surge la disyuntiva de conservar sus propias costumbres de su lugar de origen o adoptar las de la urbe receptora. Lo anterior motiva y amplía las posibilidades de investigación, ya que cada día más personas alrededor del mundo adoptan la idea de que el lugar donde residen no tiene que ser permanente. 


\section{Tópicos espaciales}

La organización espacial dentro de las ciudades es el reflejo de un esquema mental de la sociedad y, a su vez, la planificación urbana es fundamental para condicionar y moldear el comportamiento de los habitantes de una urbe. Diversos factores intervienen en la planificación urbana, siendo el tema de la vivienda el más estudiado. Esto se justifica por la relevancia que representa este tópico en relación con las personas que solo van de paso por una ciudad. Por una parte están los estudios que tratan de explicar las condiciones en las que viven los grupos flotantes (Wu y Logan 2016; Jiang, 2006; Roberts, 2002) y los que abordan la percepción y satisfacción de esta población respecto de sus viviendas (Tao, Wong y Hui, 2014; Shen y Huang, 2003). Por otra parte, existen investigaciones que analizan el impacto económico en la vivienda, tanto de los pobladores temporales como de los permanentes (Wang et al.,2017; Logan, Fang y Zhang, 2009). En todos estos trabajos de alguna u otra manera se destaca que estos pobladores se encuentran en una clara desventaja con respecto a los residentes permanentes y, sin embargo, esto no es razón para volver a su ciudad de origen.

En cuanto al desarrollo y planeación urbana, el principal reto es incluir a estos pobladores como un hecho en las ciudades contemporáneas y futuras, ya que son un factor esencial para lograr un desarrollo social sustentable (Wu et al., 2016). Otro factor que juega un papel vital en los flujos poblacionales es la infraestructura, pues entre más conexiones se construyan entre las urbes mayor será el flujo (Yue et al., 2005). Siendo el equipamiento urbano un elemento fundamental dentro de toda ciudad, es relevante destacar que no se encontró ningún estudio que analice el impacto de los pobladores flotantes en relación con estos servicios, quedando en evidencia la falta de estudios al respecto que proporcionen datos cuantitativos. Resulta de sumo interés investigar acerca de cómo estos actores hacen uso de un territorio respecto del cual no fue contemplada su presencia.

\section{Enfoque del estudio socioespacial}

Como ya se dijo anteriormente para el estudio de estas poblaciones flotantes y la ciudad, se debe tener como principal reto el reconocimiento de este segmento de personas como parte de la ciudadanía, independientemente del tiempo que pasen en un territorio específico. Para ello, en la Figura $\mathrm{N}^{\circ} 2$ se muestra la ruta que se debe de seguir para garantizar la integración de estos moradores efímeros, sin importar lo complejo que esto pudiera resultar. Este es un tema que empieza a tomar relevancia a nivel internacional, debido a los vertiginosos cambios demográficos a nivel global que se viven en la actualidad, determinados por factores como conflictos bélicos, crisis económicas, persecuciones religiosas, desastres naturales, entre otros, que traen como resultado los flujos poblacionales (Panaia, 2010). 
Figura $N^{\circ}$ 2: Ruta a seguir para la integración de las poblaciones flotantes

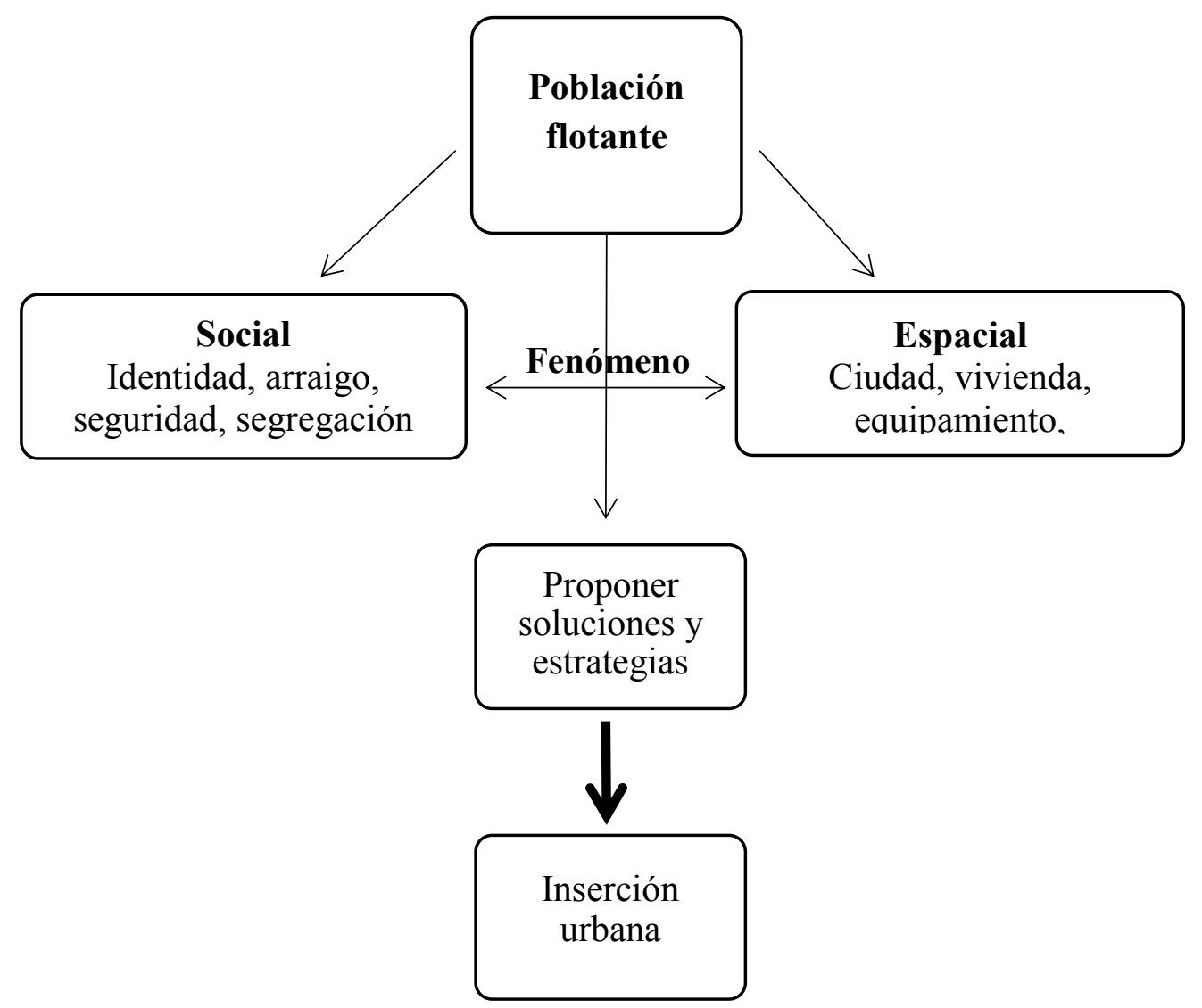

Fuente: elaboración propia.

\section{Futuros estudios}

El desafío que enfrentan los futuros estudios que aborden el tema de las poblaciones flotantes, es la aceptación del término a nivel global, puesto que no todos los estudios separan a este grupo de población de los migrantes. Además, es indispensable la generación de métodos cualitativos y cuantitativos, tal como propone Meeus (2012), que permitan comprender en profundidad el fenómeno social para de esta manera medir el impacto en las ciudades por las que transitan. Por último, es imperioso contar con estudios de esta índole alrededor del mundo para poder comparar las distintas realidades y así poder identificar similitudes y diferencias.

\section{Conclusión}

Este artículo revisa temáticas relacionadas con la investigación de las poblaciones flotantes y su interacción con las ciudades que habitan o transitan. En los cuarenta trabajos 
seleccionados, se analizaron aspectos socioespaciales y subtemas que se interrelacionan con cada uno de estos aspectos. Paulatinamente este tema ha cobrado mayor relevancia, evidenciada en la publicación creciente de investigaciones durante la última década, las que colaboran al entendimiento de este segmento de la población, así como sus variables y singularidades.

La discusión del documento identifica las áreas ya estudiadas como: identidad, segregación, arraigo, ciudad, vivienda e infraestructura. También examina áreas de oportunidad para profundizar en investigaciones futuras, tales como equipamiento urbano y seguridad. Asimismo, recoge futuras tendencias de investigación que deberían estar relacionadas con segmentos de la sociedad que no se encuentran en situaciones de precariedad y que tienden a ser excluidos. Esta línea de estudio se sugiere como ruta para los investigadores que pretenden reflexionar sobre estos flujos poblacionales.

A pesar de los intentos por parte de las ciencias sociales de profundizar en el tema, es necesario evidenciar y concientizar que esto es un fenómeno de la sociedad actual y no necesariamente un conflicto, ya que este trae consecuencias tanto positivas como negativas, las cuales se deben de afrontar de manera eficaz, partiendo de la realidad de que son parte de la sociedad misma. La investigación sobre el tema aún ofrece una amplia diversidad de tópicos por abordar para lograr cubrir la totalidad del fenómeno. Para entender el fenómeno de las poblaciones flotantes y su relación con la ciudad, se requiere desarrollar métodos que puedan ser utilizados en las diversas regiones del mundo, para de esta manera poder comparar e identificar las características globales del tema.

\section{Referencias bibliográficas}

Anant, S. (1966). The need of belong. Canada's Mental Health, 14, 21-27.

Bell, M. y Ward, G. (2000). Comparing temporary mobility with permanent. Tourism Geographies, 2(1), 97-107.

Bingqin, L. (2006). Floating population or urban citizens status, social provision and circumstances of rural-urban migrants in China. Social Policy \& Administration, 40(2), 174-195.

Caminha, C., Furtado, V., Pequeno, T., Ponte, C., Melo, H., Oliveira, E., y Jr. Andrade, J. (2017). Human mobility in large cities as a proxy for crime. Plos ONE, 12(2), 1-13.

Côté, J. y Levine, C. (2002). Identity Formation, Agency, and Culture. A Social Psychological Synthesis. Hillsdale, NJ: Lawrence Erlbaum.

Crang, M. y Zhang, J. (2012). Transient dwelling: trains as places of identification for the floating population of China. Social \& Cultural Geography, 13(8), 895-914.

De Martino, E. (1977). El fin del mundo. Londres: Exter. 
Duhau, E. y Giglia, A. (2008). Las reglas del desorden: habitar la metrópoli. México: Siglo Veintiuno Editores.

Duke, F. (2007). El Neohumanismo de la no ciudad. En F. Duke, Habitar la tierra (pp. 6599). Madrid: Abada Editores.

Garrocho, C. (2011). Población flotante, población en movimiento: conceptos clave y métodos de análisis exitosos. México: Consejo Nacional de Población: El Colegio Mexiquense, A.C.

Goodkind, D. y West, L. (2002). China's floating population: Definitions, data and recent findings. Urban Studies (Routledge), 39(12), 2.237-2.250.

$\mathrm{Gu}$, P. y Ma, X. (2013). Investigation and analysis of a floating population's settlement intention and environmental concerns: A case study in the Shawan River Basin in Shenzhen, China. Habitat International, 39, 170-178.

Jiang, L. (2006). Living conditions of the floating population in urban China. Housing Studies, 21(5), 719-744.

Ji-Wei, W., Zhi-Ting, C., Hong-Wei, C., Chang-Nian, W., Harada, K., Minamoto, K. y Ueda, A. (2010). Quality of life associated with perceived stigma and discrimination among the floating population in Shanghai, China: a qualitative study. Health Promotion International, 25(4), 394-402.

Ley, J. y Fimbres, N. (2011). La expansión de la ciudad de Mexicali: una aproximación desde la visión de sus habitantes. Región y Sociedad, 23(52), 210-238.

Liang, Z. y Ma, Z. (2004). China's floating population: new evidence from the 2000 census. Population \& Development Review, 30(3), 467-488.

Logan, J., Fang, Y. y Zhang, Z. (2009). Access to housing in urban China. International Journal of Urban \& Regional Research, 33(4), 914-935.

Luo, J., Zhang, X., Wu, Y., Shen, J., Shen, L., y Xing, X. (2018). Urban land expansion and the floating population in China: for production or for living? Cities, 74, 219228.

Meeus, B. (2012). How to 'catch' floating populations? Research and the fixing of migration in space and time. Ethnic \& Racial Studies, 35(10), 1.775-1.793.

Mohabir, N., Jiang, Y. y Ma, R. (2017). Chinese floating migrants: rural-urban migrant labourers' intentions to stay or return. Habitat International, 60, 101-110.

Nielsen, I., Nyland, C., Smyth, R., Mingqiong, Z. y Jiuhua Zhu, C. (2006). Effects of intergroup contact on attitudes of Chinese urban residents to migrant workers. Urban Studies (Routledge), 43(3), 475-490.

Panaia, M. (2010). Algunas precisiones sobre el concepto de población en el ámbito del trabajo. Revista Interuniversitaria de Estudios Territoriales, 6, 27-36. 
Qiao, L., Li, Y., Liu, Y. y Yang, R. (2016). The spatio-temporal change of China's net floating population at county scale from 2000 to 2010. Asia Pacific Viewpoint, 57(3), 365-378.

Quintero, M. (2008). La naturaleza de las representaciones sociales. Revista Latinoamericana de Ciencias Sociales, Niñez y Juventud, 6(1), 55-80.

Roberts, K. (2002). Female labor migrants to Shanghai: temporary 'floaters' or Potential settlers? International Migration Review, 36(2), 492-519.

Shen, J., (2013). Increasing internal migration in China from 1985 to 2005: institutional versus economic drivers. Habitat International, 39, 1-7.

Shen, J. y Huang, Y. (2003). The working and living space of the 'floating population' in China. Asia Pacific Viewpoint, 44(1), 51-62.

Tan, M., Li, X., Lu, C., Luo, W., Kong, X. y Ma, S. (2008). Urban population densities and their policy implications in China. Habitat International, 32(4), 471-484.

Tao, L., Wong, F. y Hui, E. (2014). Residential satisfaction of migrant workers in China: a case study of Shenzhen. Habitat International, 42, 193-202.

Teixeira, C. (2000). Política cultural, cultura e imaginario. Instituto Tecnológico de Estudios Superiores de Occidente. México: Consejo Nacional para la Cultura y las Artes, Secretaría de Cultura del Gobierno de Jalisco.

Walcott, S. y Pannell, C. (2006). Metropolitan spatial dynamics: Shanghai. Habitat International, 30(2), 199-211.

Wang, Y., Wang, S., Li, G., Zhang, H., Jin, L., Su, Y. y Wu, K. (2017). Identifying the determinants of housing prices in China using spatial regression and the geographical detector technique. Applied Geography, 79, 26-36.

Webber, M. (1963). Order in diversity: community without propinquity. En W. L., Cities and Space: The Future Use of Urban Land (pp. 23-54). Baltimore: Johns Hopkins University Press.

Wu, F. y Logan, J. (2016). Do rural migrants 'float' in urban China? Neighbouring and neighbourhood sentiment in Beijing. Urban Studies (Sage Publications Ltd.), 53(14), 2973-2990. doi:10.1177/0042098015598745.

Wu, Y., Luo, J., Zhang, X., y Skitmore, M. (2016). Urban growth dilemmas and solutions in China: Looking forward to 2030. Habitat International, 56, 42-51.

Yue, T., Wang, Y., Liu, J., Chen, S., Qiu, D., Deng, X. y ... Su, B. (2005). Surface modelling of human population distribution in China. Ecological Modelling, 181(4), 461-478. doi: 10.1016/j.ecolmodel.2004.06.042.

Zai, L., y Zhongdong, M. (2004). China's floating population: new evidence from the 2000 census. Population and Development Review, 30(3), 467-488. 
Zhu, Y. (2007). China's floating population and their settlement intention in the cities: beyond the Hukou reform. Habitat International, 31(1), 65-76. doi: 10.1016/j.habitatint.2006.04.002. 